ИЗВЕСТИЯ АКАДЕМИИ НАУК ЭСТОНСКОИ ССР. ТОМ 28 ФИЗИКА * МАТЕМАТИКА. 1979, № 2

\title{
ОПРЕДЕЛЕНИЕ НАПРЯЖЕНИЙ В КУБИЧЕСКИХ МОНОКРИСТАЛЛАХ ЦИЛИНДРИЧЕСКОЙ ФОРМЫ МЕТОДОМ РАССЕЯННОГО СВЕТА
}

\author{
(Представлена Х. Абеном)
}

Трехмерные напряженные состояния в кубических монокристаллах определяются неразрушающим методом интегральной фотоупругости $\left[{ }^{1-3}\right]$. Вместе с тем для изучения объемного напряженного состояния модели можно использовать и метод рассеянного света $\left[{ }^{4-9}\right]$. Теоретически этот вопрос для кристаллов рассматривался в $\left[{ }^{10}\right]$, о практических же исследованиях сведения в литературе отсутствуют. В настоящей работе область применения метода рассеянного света распространяется на изучение напряжений в кубических монокристаллах цилиндрической формы.

\section{1. Методика эксперимента}

При выращивании из расплава кристаллы находятся в осесимметричном температурном поле. Поэтому можно предполагать, что в изучаемых длинных кристаллах цилиндрической формы напряженное состояние является осесимметричным и напряжения от координаты $z$ не зависят (рис. 1). При этом кристаллографическая ось [001] совпадает с осью цилиндра $z$.

Методом, аналогичным использованному С. Р. Бхонсле и К. Е. Ворк $\left[{ }^{7}\right]$, сканируем кристалл вдоль диаметра, который совпадает с кристаллографической осью [100], пучком, параллельным оси $z$, и измеряем разность хода $\delta_{1}$. Дифференциальный закон Вертгейма имеет в данном случае вид $\left[{ }^{10,11}\right]$

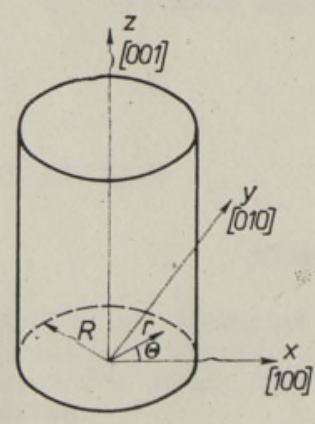

$$
\mathrm{d} \delta_{1} / \mathrm{d} z=C^{\prime}{ }_{11}\left(\sigma_{r}-\sigma_{\theta}\right),
$$

где $C_{11}{ }^{\prime}=n_{0}{ }^{3}\left(\pi_{11}-\pi_{12}\right) / 2, n_{0}-$ показатель преломления, $\pi_{i j}$ - пьезооптические коэффициенты, $\sigma_{r}$, $\sigma_{\theta}-$ радиальное и окружное напряжения соответственно.

Экспериментально напряжения можно определить с точностью до изотропного растяжения [ $\left.{ }^{8}\right]$. Для полного решения задачи введем дополнительно уравнение равновесия

Рис. 1. Координаты модели. 


$$
\partial \sigma_{r} / \partial r+\left(\sigma_{r}-\sigma_{\theta}\right) / r=0 .
$$

Интегрированием последнего уравнения с учетом соотношения (1) получаем выражение для определения напряжения $\sigma_{r}$ :

$$
\sigma_{r}=-\frac{1}{C_{11}^{\prime}} \int_{r_{0}}^{r} \frac{1}{r} \frac{\mathrm{d} \delta_{1}}{\mathrm{~d} z} \mathrm{~d} r+\sigma_{r}\left(r_{0}\right) .
$$

Напряжение $\sigma_{\theta}$ вычисляется из соотношения (1).

Для определения $\sigma_{z}$ просвечиваем кристалл вдоль диаметра, который совпадает с кристаллографической осью [100], и измеряем разность хода $\delta_{2}$ вдоль пучка. Закон Вертгейма принимает вид

$$
\mathrm{d} \delta_{2} / \mathrm{d} s=C^{\prime}{ }_{11}\left(\sigma_{\theta}-\sigma_{z}\right),
$$

откуда вычисляется напряжение $\sigma_{z}$.

- Описанный метод позволяет вычислять напряжения также в изотропных телах. Однако отметим, что необходимость просвечивания кристалла в двух направлениях - вдоль и перпендикулярно оси $z$ - вызывает технические затруднения в случае больших моделей.

Рассмотрим теперь методику определения напряжений, которая не требует просвечивания модели в направлении оси $z$. Просвечиваем кристалл не только в направлении [100], но и в направлении [110] вдоль диаметра. Выражение для разности хода $\delta_{3}$ принимает вид $[1,10]$

$$
\frac{\mathrm{d} \delta_{3}}{\mathrm{~d} r}=\frac{1}{2}\left(C_{11}^{\prime}-C_{44}\right) \sigma_{r}+\frac{1}{2}\left(C_{11}^{\prime}+C_{44}\right) \sigma_{\theta}-C_{11}^{\prime} \sigma_{z}
$$

где $C_{44}=\left(n_{0}^{3} \pi_{44}\right) / 2$.

На основе выражений (2), (4) и (5) напряжение $\sigma_{r}$ вычисляется по формуле

$$
\sigma_{r}=\frac{2}{C_{11}^{\prime}-C_{44}} \int_{r_{0}}^{r} \frac{1}{r}\left(\frac{\mathrm{d} \delta_{2}}{\mathrm{~d} r}-\frac{\mathrm{d} \delta_{3}}{\mathrm{~d} r}\right) \mathrm{d} r+\sigma_{r}\left(r_{0}\right),
$$

напряжение $\sigma_{\theta}$ - по формуле

$$
\sigma_{\theta}=\sigma_{r}+\frac{2}{C_{11}^{\prime}-C_{44}}\left(\frac{\mathrm{d} \delta_{2}}{\mathrm{~d} r}-\frac{\mathrm{d} \delta_{3}}{\mathrm{~d} r}\right),
$$

а напряжение $\sigma_{z}$ - по формуле (4).

Преимущество изложенного метода заключается в том, что кристалл следует поворачивать только вокруг оси $z$, что экспериментально легко осуществимо. Точность метода зависит от величины $C_{11}^{\prime}-C_{44}$. Однако в изотропных средах, где $C_{11}^{\prime}=C_{44}$, он неприменим, поскольку уравнение (5) не дает никакой новой информации о распределении напряжений.

\section{2. Обработка данных}

Для сглаживания экспериментальных данных представляем их в виде степенных рядов. Так как напряжения являются осесимметричными, а разности хода, следовательно, антисимметричными функциями относительно точки $r=0$, то в разложенния $\delta_{2}$ и $\delta_{3}$ сохраняем только нечет- 


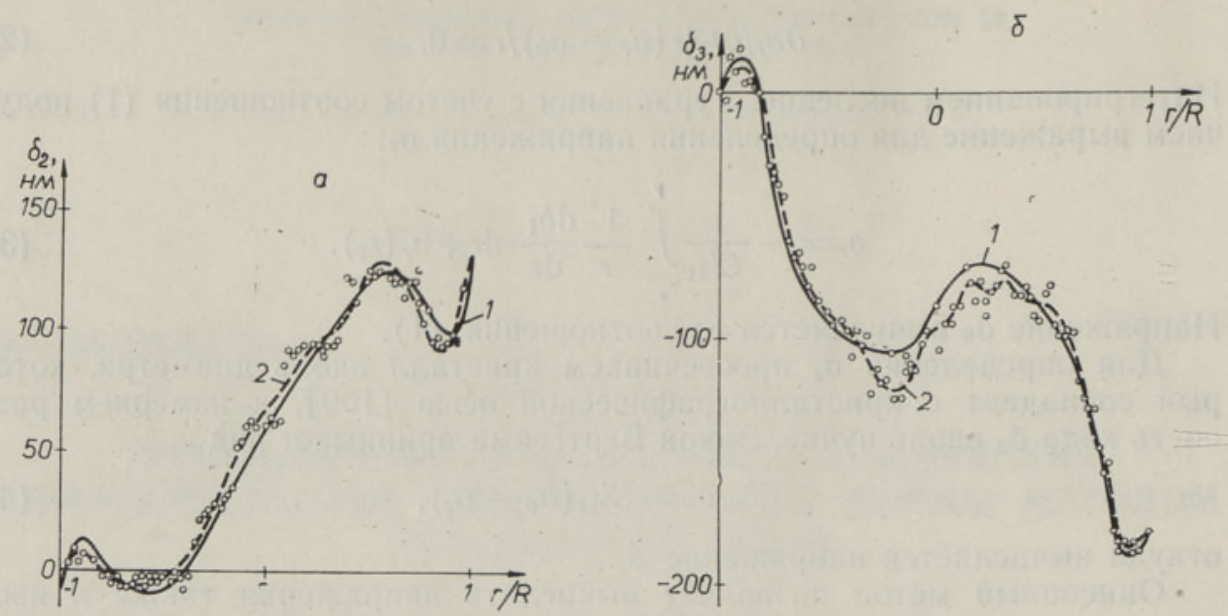

Рис. 2. Расчет разности хода вдоль диаметра в направлениях [1.100] (a) и [110] (б) с применением полиномов (кривые 1) и сплайнов (кривые 2) (О - экспериментальные данные).

ные члены. Коэффициенты в этих рядах вычисляются методом наименьших квадратов, после чего из формул (4), (6) и (7) определяются напряжения в кристалле.

Если же для описания распределения экспериментальных данных необходимо сохранить в разложении большое количество членов, тогда ошибки, возникающие при вычислении производных, могут быть весьма существенными. Чтобы избежать этого, иногда целесообразно представлять данные в виде сплайнов $\left[{ }^{12,13}\right]$. В случае использования метода рассеянного света удобны кубические сплайны. К. Х. Райнш $\left[{ }^{13}\right]$ предложил для первой и последней точек измерения условие

$$
\mathrm{d}^{2} g / \mathrm{d} x^{2}=0 .
$$

Однако по мнению Д. Г. Бергхауса и Ю. П. Каннона [ $\left.{ }^{12}\right]$, такое условие не всегда корректно и может приводить к значительным ошибкам на краях модели. Поэтому в настоящей работе экспериментальные данные в первой и последней точках измерения вначале сглаживаются степенными рядами третьего порядка, а затем вычисляются на их основе вторые производные, которые и используются вместо граничных условий (8).

\section{3. Экспериментальное исследование монокристалла $\mathrm{KCl}$}

Монокристалл $\mathrm{KCl}$ длиной $\sim 400$ мм и диаметром $\sim 80$ мм просвечивался в иммерсионной жидкости, в качестве которой использовалась смесь $\alpha$-монобромнафталина и медицинского вазелинового масла. Источником света служил лазер ЛГ-56 $(\lambda=632,8 \mathrm{mM})$, разность хода определялась компенсатором Краснова СКК-2. Интенсивность рассеянного света регистрировалась фотоумножителем ФЭУ-38, и ток последнего измерялся микровольтметром В2-11.

Фотоупругие коэффициенты $\mathrm{KCl}$ имеют следующие значения $\left[{ }^{14}\right]$ : $C_{11}^{\prime}=2,7 \cdot 10^{-7} \quad \mathrm{~cm}^{2} / \kappa 2, C_{44}=-7,3 \cdot 10^{-7} \mathrm{~cm}^{2} / \kappa 2$. Экспериментальные данные сглаживали полиномами 13-й степени и сплайнами (см. рис. 2). Условие макростатики 

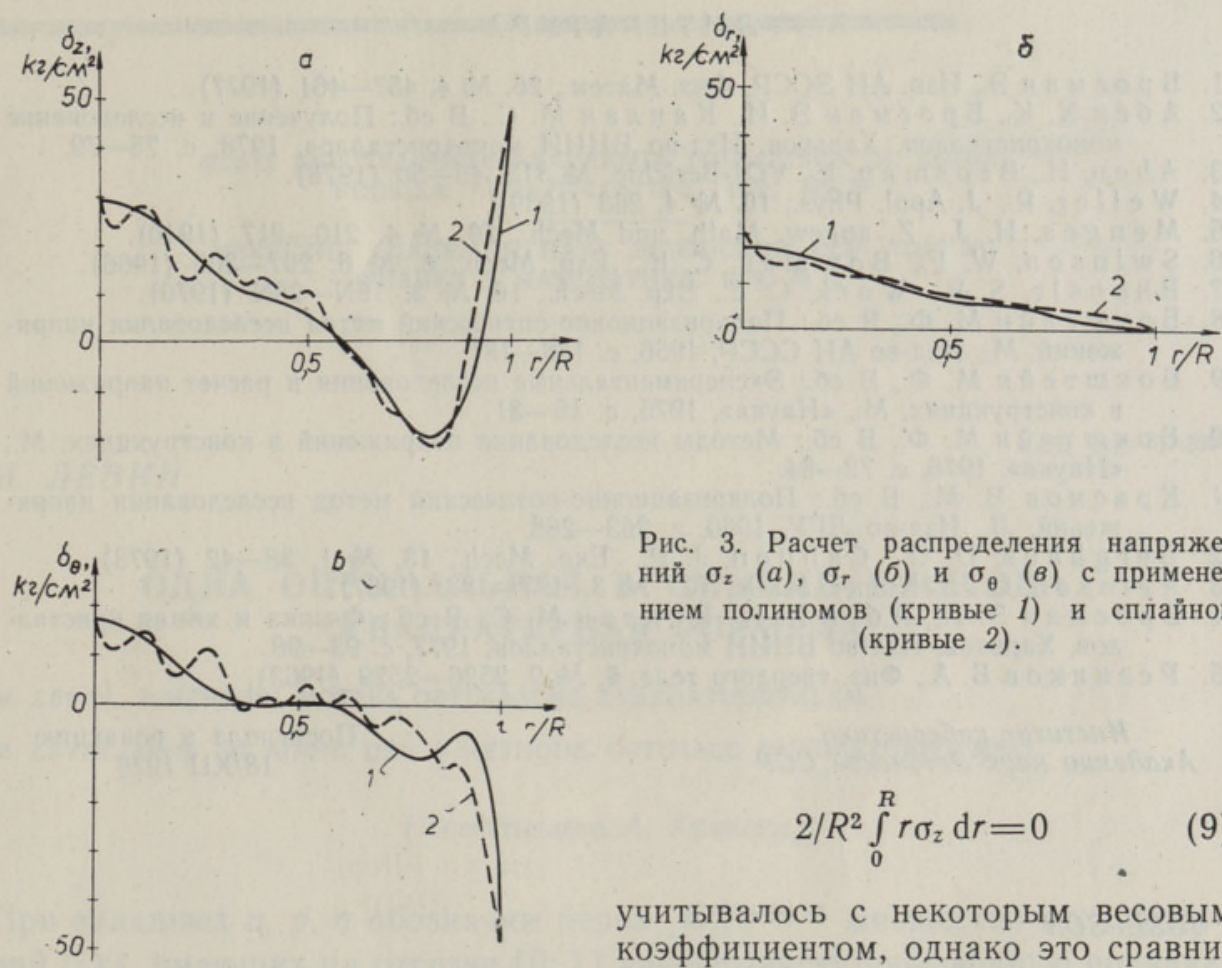

Рис. 3. Расчет распределения напряжений $\sigma_{z}(a), \sigma_{r}(б)$ и $\sigma_{\theta}($ (в) с применением полиномов (кривые 1) и сплайнов (кривые 2).

$$
2 / R^{2} \int_{0}^{R} r \sigma_{z} \mathrm{~d} r=0
$$

учитывалось с некоторым весовым коэффициентом, однако это сравнительно мало повлияло на величины вычисляемых напряжений.

На рис. 3 показаны графики напряжений, полученные с помощью полиномов и сплайнов. При использовании степенных рядов условие макростатики (9) выполняется с точностью до $1 \%$ от среднего напряжения $\sigma_{z}$.

Из рис. $3, a$ видно, что распределение $\sigma_{z}$ не является параболическим. Возможность появления такого «аномального» распределения напряжений теоретически предсказана Б. А. Резниковым $\left[{ }^{15}\right]$ и экспериментально доказана методом интегральной фотоупругости Х. Абеном и Э. Бросманом :[3].

Из рис. 3 видно также, что сплайны довольно хорошо описывают не только распределение разности хода, но и существующие в кристалле локальные неоднородности, которые присваиваются всему кристаллу.

\section{Выводы}

1. На примере кристалла $\mathrm{KCl}$ показано, что метод рассеянного света может быть успешно применен при исследовании напряжений в кубических монокристаллах цилиндрической формы. При этом предложенная методика эксперимента отличается простотой благодаря тому, что кристалл просвечивается лишь в двух направлениях поперечного сечения - [100] и [110].

2. Обработка экспериментальных данных с помощью разработанной нами методики степенных рядов и сплайнов подтвердила выводы других исследователей о том, что распределение осевых напряжений в кристаллах не всегда является параболическим. 


\section{ЛИТЕРА Т Р РА}

1. Б р ос м а н Э. Изв. АН ЭССР, Физ. Матем., 26, № 4, $457-461$ (1977).

2. А бе н Х. К., Б р ос м а Н. И., К а п л а М. С., В сб.: Получение и исследование монокристаллов, Харьков, Изд-во ВНИИ монокристаллов, 1978, с. 75-79.

3. A be n. H.. B rosm a n. E., VDI-Berichte. № 313, 45-50 (1978).

4. W e 11 e r, R., J. Appl. Phys., 10, № 4, 266 (1939).

5. Me n ges, H. J., Z. angew. Math. und Mech., 20, № 4, 210-217 (1940),

6. Swinson, W. F., B ow m a n, C. E., Exp. Mech., 6, № 6, 297-305 (1966).

7. B honsle, S. R., Work, C. E., Exp. Mech., 10, № 3, 19N-28N (1970).

8. Бок ш т й н М. Ф., В сб.: Поляризационно-оптический метод нсследования напряжений, М., Изд-во АН СССР, 1956, с. 138-181.

9. Бок ш т й н М. Ф., В сб.: Экспернментальные исследования и расчет напряжений в конструкциях, М., «Наука», 1975 , с. $18-31$.

10. Бок ш те й н М. Ф., В сб.: Методы исследования напряжений в конструкциях, М., «Наука», 1976 , с. $72-84$.

11. К р аснов В. М., В сб.: Поляризашионно-оптический метод исследования напряжений, Л.. Изд-во ЛГУ, 1960. с. 263-268.

12. Berghaus, D. G., C a n n o n, J. P., Exp. Mech., 13, № 1, 38-42 (1973).

13. Reins ch, C. H., Numer. Math., 10, № 3, 177-183 (1967).

14. Бросм ан Э. И., А бен Х. К.. К ап лан М. С., В сб.: Физика и химия кристаллов, Харьков, Изд-во ВНИИ монокристаллов, 1977, с. 93-98.

15. Р ез н и ко в Б. А., Физ. твердого тела, 5, № 9, 2526-2529 (1963).

Институт кибернетики

Академии наук Эстонской ССР
Поступила в редакцию 18/XII 1978

\section{J. JOSEPSON}

\section{HAJUTATUD VALGUSE MEETODI KASUTAMINE PINGETE MĂARAMISEKS SILINDRILISE KUJUGA KUUBILISTES MONOKRISTALLIDES}

Artiklis on kirjeldatud pikkades silindrilistes monokristallides esinevate pingete määramise metoodikat eeldusel, et puudub pingete gradient piki $z$-telge. Sel juhul piisab, kui valgustada kristalli risti $z$-teliega piki diameetrit paralleelselt kristallograafiliste telgedega [100] ja [110] ning mōōta käiguvahet piki valguskiiri. Katseandmete silumiseks on kasutatud polünoome ja splaine. Katseliselt on mõõdetud pingeid ühes $\mathrm{KCl}$ monokristallis.

\section{J. JOSEPSON}

\section{STRESS DISTRIBUTION IN CUBIC SINGLE CRYSTALS OF CYLINDRICAL FORM BY SCATTERED-LIGHT PHOTOELASTICITY}

This paper reports the use of scattered-light photoelasticity to determine the stress distributions in cubic single crystals of cvlindrical form. It is assumed that there is no stress gradient along the cylinder axis. That is the case in the middle part of comparatively long single crystals. The retardation is measured if the light nasses nerpendicular to the $z$-axis along the diameter parallel to the crystallographic axis [100] and [110]. The principal stresses are separated using the equation of equilibrium.

The stresses in a $\mathrm{KCl}$ cylinder are determined. The distributions of retardations along the diameter are shown in figures. The data points are fitted to polynomials and to smoothed splines. Least squares curve fits can yield erratic derivative values when high-order polynomials are used, but they permit to use symmetrical assumptions about the retardation distribution. The smoothed cubic spline is used for obtaining highquality derivatives from experimental data, but splines give an exact description of the local heterogeneity of the material.

It is seen that the distribution of the axial stress deviates from a simple parabola. Such an «anomalous» stress distribution has been theoretically predicted and has been experimentaly measured by the integrated photoelasticity. 